\title{
Performance of shape memory composite with SMA and SMP
}

\author{
H. Tobushi ${ }^{1, a}$, S. Hayashi ${ }^{2, b}$, Y. Sugimoto ${ }^{1, c}$ and K. Date D, d $^{1,}$ \\ ${ }^{1}$ Department of Mechanical Engineering, Aichi Institute of Technology, \\ 1247 Yachigusa, Yakusa-cho, Toyota, 470-0392, Japan. \\ ${ }^{2}$ DiAPLEX Co., Ltd., Ebisu-minami 1-5-2, Shibuya-ku, Tokyo, 150-0022, Japan

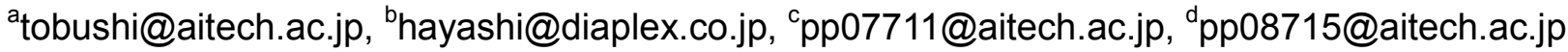

Keywords: Composite, Shape Memory Alloy, Shape Memory Polymer, Shape Fixity, Shape Recovery, Recovery Force, Bending

\begin{abstract}
The shape-memory composite belt with a TiNi-SMA wire fiber and a polyurethane-SMP sheet matrix was fabricated. The bending actuation characteristics of the belt were investigated by the thermomechanical tests. Residual deflection obtained by cooling under constant maximum deflection is close to the maximum deflection. The residual deflection disappears by heating under no load. Both the rates of shape fixity and shape recovery are close to $100 \%$. Recovery force which appears by heating under constant residual deflection is $93-94 \%$ of the maximum force. The model of bending-spring constant of the shape-memory composite belt is proposed and evaluated based on the experimental data.
\end{abstract}

\section{Introduction}

Shape memory materials (SMMs) have been developed as smart materials. In SMMs, shape memory alloy (SMA) and shape memory polymer (SMP) have been used in practical applications ${ }^{[1]}$. The shape memory effect (SME), superelasticity (SE) and large recovery stress appear in SMA ${ }^{[2],[3]}$. However, both elastic modulus and yield stress are low at low temperature. This means the fact that rigidity of SMA elements is low at low temperature. The shape fixity (SF) and large shape recovery (SR) appear in $\mathrm{SMP}^{[4]-[6]}$. However, both elastic modulus and yield stress are low at high temperature. This means the fact that rigidity of SMP elements is low at high temperature. If the shape memory composites (SMCs) with SMA and SMP are developed, new and higher functionality of the material can be obtained. That is, shape fixity and high rigity at low temperature and shape recovery and recovery stress at high temperature can be used in the SMC. In the present paper, the characteristics of the SMC belt with SMA and SMP and the bending actuation properties were analysed. A TiNi SMA wire was used for the fiber of the SMC. A polyurethane (PU) SMP sheet was used for the matrix of the SMC. Since the fatigue life of TiNi SMA is very long, it has been most widely used in SMAs. Since PU SMP has various kinds of properties, it has been used in practical applications. The model of bending-spring constant of the SMC belt was proposed. 


\section{Characteristics of composite with SMA and SMP}

The relationships between elastic modulus and temperature and those between yield stress and temperature for TiNi SMA, PU SMP and mild steel are schematically shown in Fig. 1. As it can be seen, the dependence of elastic modulus and yield stress on temperature is quite different among SMA, SMP and steel. In order to obtain the two-way shape memory effect (TWSME), two-way movement of SMA elements, the combination of SMA and bias materials, for example, steel is used in practical applications ${ }^{[1]-[3]}$. If the composite materials with these materials are developed, new properties can be achieved. For example, if SMA and SMP are combined, the composite material with large recovery strain, high recovery stress, high rigidity, TWSME and light weight can be obtained. Therefore, if the SMCs with SMA and SMP are developed, new and higher functionality of the material can be expected.

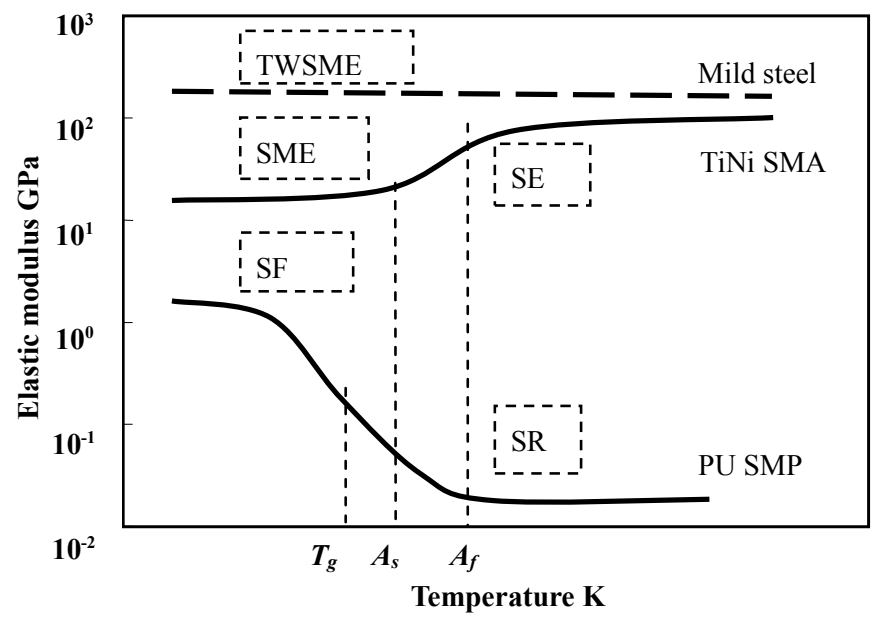

(a) Elastic modulus

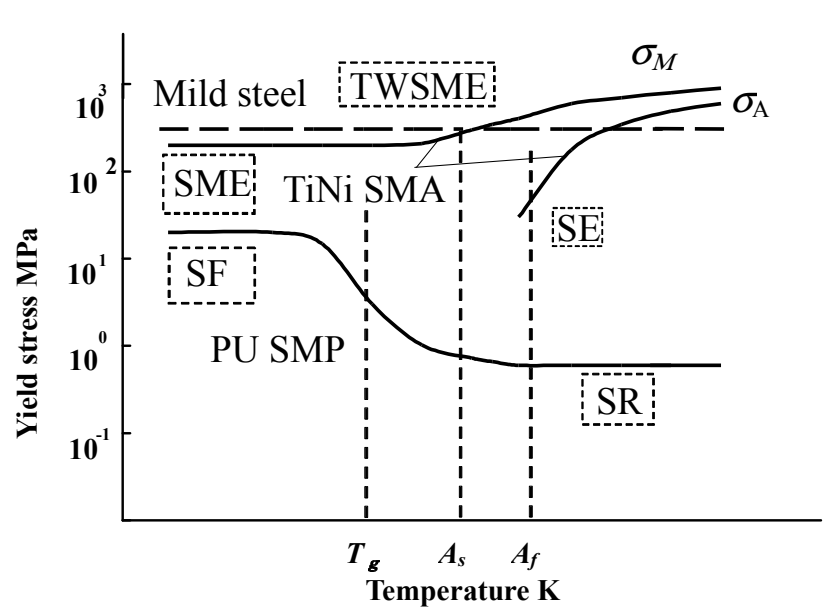

(b) Yield stress

Fig. 1 Dependence of elastic modulus and yield stress on temperature

\section{Materials and Specimen}

Polyurethane SMP (Diary MP5510 produced by Mitsubishi Heavy Industries, Ltd.) was used as the matrix of the SMC belt. The glass transition temperature of the SMP sheet $T_{\mathrm{g}}$ was $328 \mathrm{~K}$. A TiNi SMA wire (produced by Furukawa Electric Co.) was used for the fibers in the SMC. Diameter and length of the wire were $0.75 \mathrm{~mm}$ and $50 \mathrm{~mm}$, respectively. The finish temperature of reverse transformation $A_{f}$ of the wire was $325 \mathrm{~K}$. The SMC belt was fabricated as follows. First the lower half of the SMP sheet with thickness of $2.1 \mathrm{~mm}$ was molded by mixing two kinds of liquid in a block. In a few minutes after molding, viscosity of the SMP became high enough to support the SMA wire on the surface of the SMP sheet. SMA wires were put on the lower half of the SMP sheet. The upper half of SMP was molded again on them. The SMC sheet was obtained by curing. Specimens of the SMC belt were cut from the SMC sheet into width of $10 \mathrm{~mm}$. The photograph of a specimen is shown in Fig. 2. As can be seen, the SMA wire was located in the central part of the SMC belt. Width, thickness and length of the SMC-belt specimen were $10 \mathrm{~mm}, 4.2 \mathrm{~mm}$ and $60 \mathrm{~mm}$, respectively. 


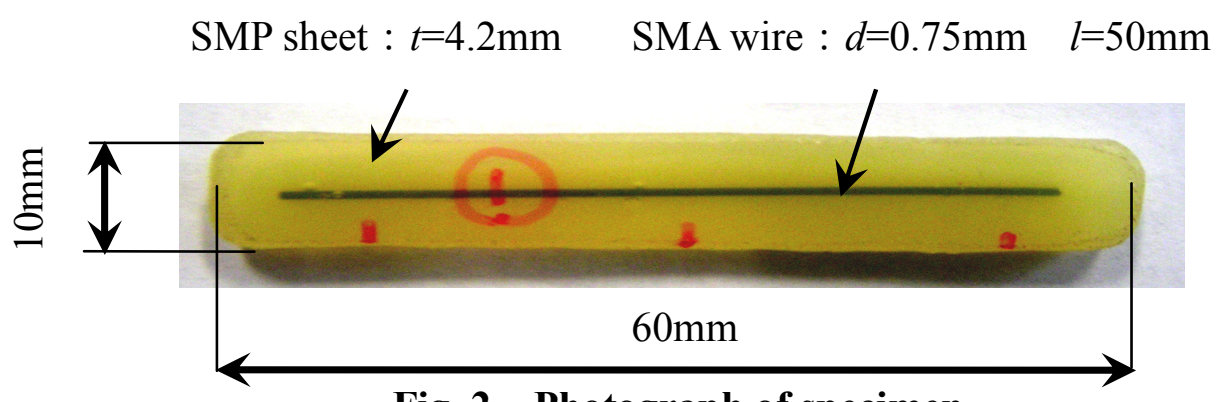

Fig. 2 Photograph of specimen

\section{Evaluation of Elastic Modulus and Spring Constant}

If a concentrated force $W$ is applied at the center of a simply supported beam, the maximum deflection $y$ is expressed by the following equation

$$
y=\frac{W l^{3}}{48 E I}
$$

where $E, I$ and $l$ denote elastic modulus, second moment of area and distance between two supports. The relationship between $W$ and $y$ is expressed by using the bending-spring constant $k$ as follows

$$
W=k \cdot y
$$

In the case of a SMA wire of diameter $d$, by using second moment of area $I_{a}=\pi \mathrm{d}^{4} / 64$, the bending-spring constant $k_{a}$ is given

$$
k_{a}=\frac{3 \pi}{4 l^{3}} E_{a} \cdot d^{4}
$$

where $E_{a}$ denotes elastic modulus of the SMA.

In the case of a SMP beam of a rectangular cross section with width $b$ and height $t$, by using second moment of area $I_{p}=b t^{3} / 12$, the bending-spring constant $k_{p}$ is expressed by the following equation

$$
k_{p}=\frac{4}{l^{3}} E_{p} \cdot b t^{3}
$$

where $E_{p}$ represents elastic modulus of the SMP.

In the present study, since the volume fraction of the SMA wire in the SMC belt is $1.05 \%$, the bending-spring constant of the SMC belt $k_{\mathrm{c}}$ can be evaluated by a sum of $k_{a}$ and $k_{p}$ as follows

$$
k_{c}=k_{a}+k_{p}=\frac{1}{l^{3}}\left(\frac{3}{4} \pi E_{a} d^{4}+4 E_{p} b t^{3}\right)
$$

With respect to the SMC belt, the bending-spring constant $k_{\mathrm{c}}$ is obtained from a slope of the initial loading curve by using Eq. (2). The SMC belt has a rectangular cross section of width $b$ and height $t$. Therefore, elastic modulus in bending of the SMC belt $E_{\mathrm{c}}$ is given by the following equation

$$
E_{c}=\frac{k_{c}}{4 b}\left(\frac{l}{t}\right)^{3}
$$


The experimental and calculated results of $k_{\mathrm{c}}$ and $E_{c}$ are shown in Table 1. As it can be seen, the bending-spring constant $k_{\mathrm{c}}$ and elastic modulus $E_{c}$ of the SMC belt can be evaluated by the proposed model. The value of elastic modulus of the SMC belt $E_{\mathrm{c}}$ at $T_{\mathrm{g}}+30 \mathrm{~K}$ is $48 \mathrm{MPa}$ which is larger than that for SMP $E_{p}=30 \mathrm{MPa}$ at $T_{\mathrm{g}}+30 \mathrm{~K}$. This means the fact that higher rigidity of SMC elements than one of SMP elements can be obtained in practical applications. As it can be found from Eq. (5), the rigidity of the SMC belt depends stronglly on diameter of the SMA fiber and height of the SMP beam.

Table1 Values of elastic modulus and spring constant

\begin{tabular}{|c|c|c|c|c|c|c|c|}
\hline \multirow{2}{*}{ Temperature } & \multicolumn{3}{|c|}{ Experimental value } & \multicolumn{4}{c|}{ Calculated value } \\
\cline { 2 - 8 } & $E_{p}[\mathrm{MPa}]$ & $E_{a}[\mathrm{GPa}]$ & $k_{c}[\mathrm{~N} / \mathrm{m}]$ & $k_{p}[\mathrm{~N} / \mathrm{m}]$ & $k_{a}[\mathrm{~N} / \mathrm{m}]$ & $k_{c}[\mathrm{~N} / \mathrm{m}]$ & $E_{c}[\mathrm{MPa}]$ \\
\hline$T_{\mathrm{g}}-25 \mathrm{~K}$ & 1150 & 20 & $\mathbf{5 5 5 0 0}$ & $\mathbf{5 3 2 5 0}$ & 233 & 53483 & 1155 \\
\hline$T_{\mathrm{g}}+30 \mathrm{~K}$ & $\mathbf{3 0}$ & $\mathbf{7 0}$ & $\mathbf{2 2 4 0}$ & $\mathbf{1 3 8 9}$ & $\mathbf{8 1 5}$ & $\mathbf{2 2 0 4}$ & 48 \\
\hline
\end{tabular}

\section{Shape Fixity and Shape Recovery}

The relationship between force and deflection and that between deflection and temperature obtained from the shape fixity and recovery test by the three-point bending with span $l=40 \mathrm{~mm}$ are shown in Fig.3.

As it can be seen (Fig. 3a), the maximum force appears at deflection of $y_{\max }=10 \mathrm{~mm}$ in the loading process at $T_{\mathrm{g}}+30 \mathrm{~K}$ (1). Force decreases almost to zero during the cooling process (2) under constant $y_{\max }$. As it can be seen in Fig. 3(b), deflection decreases at temperatures in the vicinity of $A_{f}$ and $T_{g}$ during the heating process (4) and approaches to zero at $T_{\mathrm{g}}+30 \mathrm{~K}$.

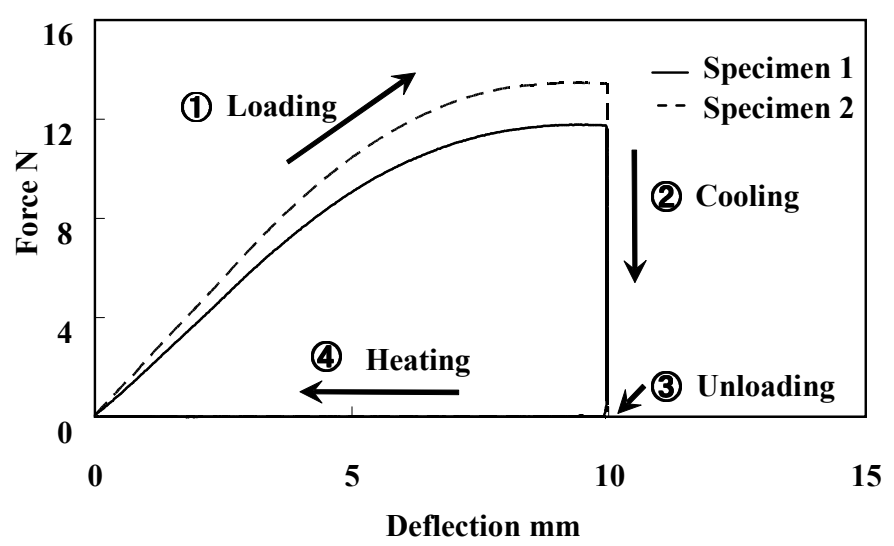

(a) Relationship between force and deflection

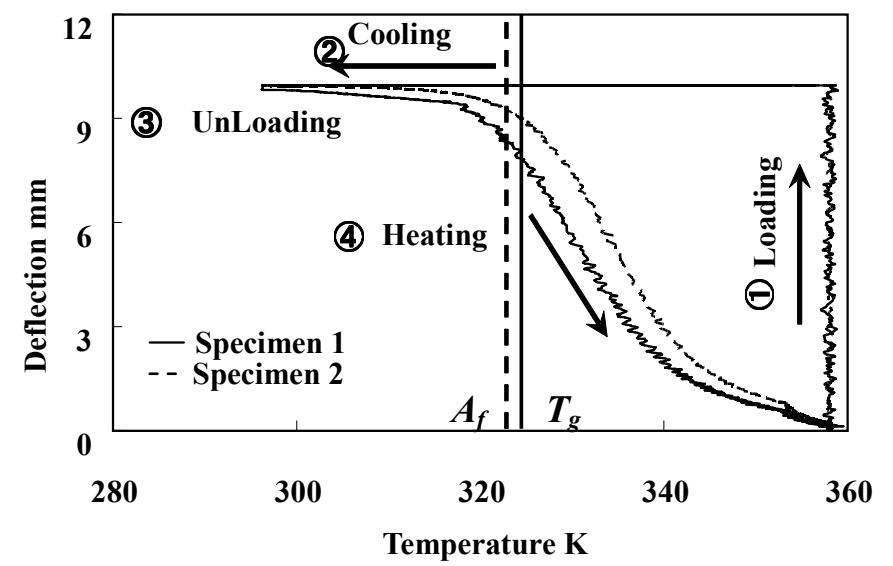

(b) Relationship between deflection and temperature

Fig. 3 Results obtained from shape fixity and recovery test by the three-point bending

Table2 Values of shape fixity and shape recovery rates

\begin{tabular}{|l|c|c|}
\hline & Shape fixity rate $R_{f}[\%]$ & Shape recovery rate $R_{r}[\%]$ \\
\hline \hline Specimen 1 & 98.7 & 98.8 \\
\hline Specimen 2 & 99.7 & 99.6 \\
\hline
\end{tabular}


The rate of shape fixity $R_{f}$ and the rate of shape recovery $R_{r}$ are defined by the following equations, respectively

$$
\begin{aligned}
& R_{f}=\frac{y_{u}}{y_{\text {max }}} \\
& R_{r}=\frac{y_{\text {max }}-y_{h}}{y_{\text {max }}}
\end{aligned}
$$

where $y_{u}$ and $y_{h}$ represent the deflection after unloading at $T_{\mathrm{g}}-30 \mathrm{~K}$ and the deflection after heating up to $T_{\mathrm{g}}+30 \mathrm{~K}$, respectively. The values of the rate of shape fixity $R_{f}$ and the rate of shape recovery $R_{r}$ are shown in Table 2. It can be seen that both $R_{f}$ and $R_{r}$ are close to $100 \%$, proving the excellent characteristics of shape fixity and shape recovery. The difference of shape fixity and shape recovery between two specimens is small.

\section{Recovery Force}

The relationship between force and deflection and that between force and temperature obtained from the recovery force test by the three-point bending with span $l=40 \mathrm{~mm}$ are shown in Fig. 4 .

As it can be seen in Fig. 4(a), the force-deflection curves during the loading (1) and cooling (2) are almost the same as those for the shape fixity and recovery test (2) observed in Fig. 3. Recovery force increases during the heating (4) under constant $y_{\max }$.

As it can be seen in Fig. 4(b), force decreases almost to zero during the cooling (2). In the heating (4), force increases significantly at temperatures in the vicinity of $A_{f}$ and $T_{\mathrm{g}}$. The value of maximum recovery force obtained by heating up to $\mathrm{Tg}+30 \mathrm{~K}$ is close to the maximum force given in the loading (1). The values of recovery force obtained during heating up to $T_{\mathrm{g}}+30 \mathrm{~K}$ and the rate of recovery force to the maximum force during the loading (1) are shown in Table 3. The rate of recovery force is $93-94 \%$. The value of recovery force is higher than $7 \mathrm{~N}$ which was obtained in the SMP belt without the SMA wire. Therefore, high recovery force can be obtained by SMC elements. The recovery force of SMC elements depends on the volume fraction and the arrangement of SMA fibers.

The development of SMC elements with high functionality is expected by various combinations of SMAs and SMPs. That is, if $A_{f}$ of SMA is higher than $T_{g}$ of SMP, the two-way property can be used during heating and cooling.

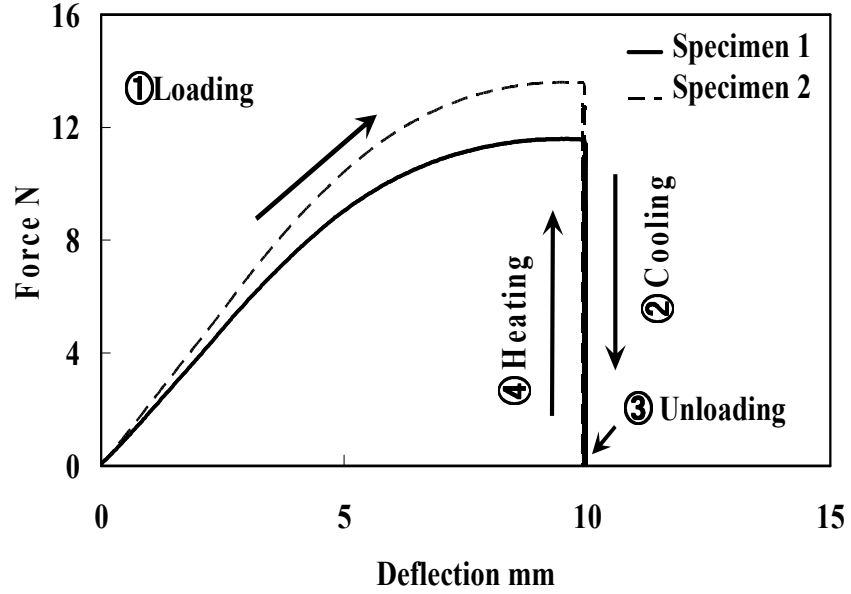

(a) Relationship between force and deflection

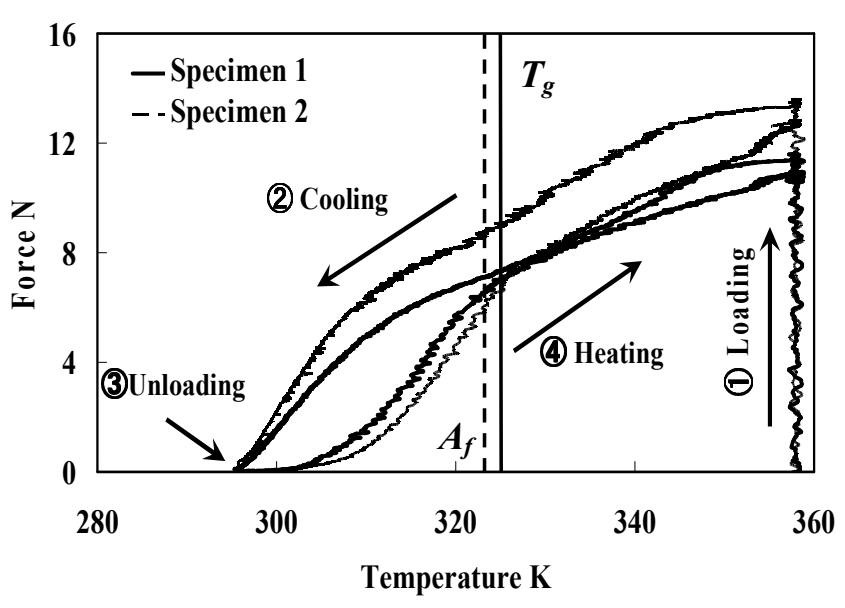

(b) Relation between force and temperature

Fig. 4 Results obtained from recovery force test by the three-point bending 
Table3 Values of maximum force, recovery force and the rate of recovery force to maximum force

\begin{tabular}{|l|c|c|c|}
\hline & Maximum force [N] & Recovery force [N] & Rate of recovery force [\%] \\
\hline \hline Specimen 1 & 11.6 & 10.9 & 93.2 \\
\hline Specimen 2 & 13.6 & 12.8 & 94.3 \\
\hline
\end{tabular}

\section{CONCLUSION}

The SMC belt was fabricated by combining the TiNi-SMA wire fiber and the polyurethane-SMP sheet matrix. The bending actuation characteristics of the SMC belt were investigated. The results obtained can be summarized as follows.

(1) Although spring constant of the SMC belt for bending is large at low temperature, it is small at high temperature. The spring constant of the SMC belt can be evaluated by the proposed model with simple combination of the SMA wire and the SMP sheet.

(2) Force decreases on cooling under constant maximum deflection and high residual deflection close to the maximum deflection is obtained. The residual deflection disappears on heating under no load. The rates of shape fixity and shape recovery close to $100 \%$ can be obtained.

(3) The recovery force which appears on heating under constant residual deflection is $93-94 \%$ of the maximum force given in the loading process at $T_{\mathrm{g}}+30 \mathrm{~K}$.

\section{References}

[1] M. Mertmann (ed) : Proc. Int. Conf. on Shape Memory and Superelastic Technologies, (ASM Inter., 2006), pp. 501-642.

[2] H. Funakubo (ed) : Shape Memory Alloys, (Gordon and Breach Science Pub., NewYork. 1987).

[3] K. Otsuka. and C. M. Wayman (ed) : Shape Memory Materials, (Cambridge University Press, Cambridge. 1998).

[4] S. Hayashi., "Properties and Applications of Polyurethane Series Shape Memory Polymer", Int. Progr. Urethanes, Vol. 6 (1993): pp. 90-115.

[5] H. Tobushi, T. Hashimoto, N. Ito, S. Hayashi and E. Yamada., "Shape Fixity and Shape Recovery in a Film of Shape Memory Polymer of Polyurethane Series", J. Intell. Mater. Syst. Struct., Vol. 9 (1998): pp. 127-136.

[6] H. Tobushi, D. Shimada, S. Hayashi and M. Endo., "Shape Fixity and Shape Recovery of Polymer Foams", Proc. Instn. Mech. Engrs., Part L.: J. Materials: Design and Applications, Vol. 217 (2003): pp. 135-143. 